\title{
A hybrid control strategy for vibration damping and precise tip-positioning of a single-link flexible manipulator
}

\author{
Emiliano Pereira*, Sumeet S Aphale ${ }^{\dagger}$, Vicente Feliu* and S O Reza Moheimani ${ }^{\ddagger}$ \\ ${ }^{*}$ ETS Ingenieros industriales, University of Castilla-La Mancha, Ciudad Real, Spain \\ Email: emiliano.pereira@uclm.es and vicente.feliu@uclm.es \\ ${ }^{\dagger}$ Centre for Applied Dynamics Research, University of Aberdeen, Aberdeen, Scotland, UK \\ Email: s.aphale@abdn.ac.uk

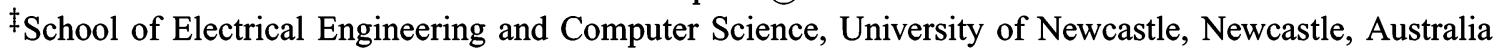 \\ Email: reza.moheimani@newcastle.edu.au
}

\begin{abstract}
In this work, we propose a new control approach for a single-link flexible manipulator, based on the Integral Resonant Control (IRC) scheme. A hybrid control scheme consisting of two nested loops by treating the joint angle and the torque measured at the base of the arm (coupling torque) as the system outputs are formulated. It is shown that the IRC scheme, a high performance controller design methodology for flexible structures with collocated actuator-sensor pairs, can be implemented in a flexible manipulator to achieve precise end-point positioning with effective vibration suppression. Experimental results are presented in order to validate the proposed control scheme. Finally, a brief discussion is included to highlight the contributions of this work in broad area of controlling single-link flexible manipulators.
\end{abstract}

\section{INTRODUCTION}

The past two decades have seen significant interest being generated in the control of flexible robotic manipulators. Novel robotic applications demand lighter robots that can be driven using small amounts of energy, for example: robotic booms in the aerospace industry, where lightweight robot manipulators with high performance requirements (high speed operation, better accuracy) are demanded [1]. Unfortunately, the flexibility of these robotic arms leads to oscillatory behavior at the tip of the link, making precise pointing or tip positioning a daunting task that requires complex closed-loop control. In order to address control objectives, such as tip position accuracy and suppression of residual vibration, many control techniques have been applied to flexible robots (see, for instance, the surveys of Benosman and Vey [2] and Dwivedy Eberhard [3] or the book written by Fraser and Daniel [4]).

There are two main problems that complicate the control design for flexible manipulators viz: (i) the high order of the system and (ii) the nonminimum phase dynamics that exist between the tip position and the input (torque applied at the joint). These problems have motivated researchers to employ in a wide range of control design techniques such as linear quadratic gaussian (LQG) [5], linear quadratic regulator (LQR) [6], pole placement [7], and inverse dynamics based control [8]. The drawback of these model-based approaches is that the complexity of the control algorithm increases significantly with the system order and the stability of the closed-loop system is sensitive to (a) changes in the robot payload, (b) model parameter uncertainties and (c) highorder unmodeled dynamics as the control bandwidth is raised (spillover effects [9]). In order to address these problems, solutions based on adaptive control [10], $H_{\infty}$ control [11], $\mu$-synthesis [12], sliding-mode control [13], neural networks [14] and fuzzy logic algorithms [15] have been investigated. However, the complex design methodologies necessitated by these techniques make their application to flexible robotics less attractive.

The use of alternative outputs has emerged as a potential solution to the problem of nonminimum phase dynamics. In [16], an alternative output, the so-called reflected tip position, was proposed. It was demonstrated therein that the transfer function from the motor torque to the reflected tip velocity is passive. Therefore, strictly passive controllers make the system stable in $L_{2}$ sense. However, the passive relationship depends on the value of the hub inertia, which must be sufficiently small in relation with beam inertia. Liu and Yuan [17] proposed an additional control loop to make the passive relationship independent of the system parameters. However, the main limitation of these passivity based control schemes is that they make the system very sensitive to joint frictions. Other solutions based on two control loops can be found in [18], [19] and [20]. The inner loop, which takes a collocated input-output, is used to damp the vibrations whereas the outer loop is used for position control. However, in [18] and [19] the stability of the position control depends on the link and motor parameters, which complicates the design. Although the vibration damping proposed in [20] was quite efficient when a motor without reduction gear was used, the residual vibration suppression is not effective when reduction gears are employed.

In [21], a new methodology for passivity based control with two nested loops was proposed. In this design, the inner loop is the position controller, which only depends on the motor 
dynamics, and the outer loop always guarantees an effective vibration suppression (with or without the reduction gear) and the stability is independent of the link parameters. This technique has some disadvantages like high sensitivity at low frequencies that complicates its implementation on a practical experimental platform (offset in the sensors, gravity effect). It also suffers from instability issues due to antialising filters.

This work builds on the control methodology of [21] and proposes a new approach based on the Integral Resonant Control (IRC) scheme [22]. The IRC scheme results in an easyto-tune, low-order damping controller, that imparts substantial damping to multiple resonant modes, without instability issues due to unmodeled system dynamics [22]. In this work we retain the advantages of [21] and alleviate the instability problems due to high sensitivity at low frequencies and antialising filters by using the IRC scheme as our choice of damping controller.

\section{A. Organization}

The paper is organized as follows. Section II provides a description of the experimental setup. The system model and the associated parameters are briefly explained in Section III. Section IV gives the details of the general control scheme. A practical implementation of the proposed control scheme on a single-link flexible robot and the obtained experimental results are presented in Section V. Section VI concludes the paper.

\section{EXPERIMENTAL SETUP}

Figure 1 shows a photograph of the experimental singlelink flexible manipulator used as the experimental platform in this work. The setup consists of (a) a DC motor (Maxon Motor EC-60) with a Harmonic Drive reduction gear 1:50 (HFUC-32-50-20H), (b) a flexible single-link comprised of a slender aluminum beam with a rectangular cross section that is attached to the motor hub in such a way that it rotates only in the horizontal plane, so that the effect of gravity can be ignored and (c) a mass with negligible inertia at the end of the arm. In addition, two sensors are used: 1) an encoder mounted at the joint of the manipulator to measure the motor angle, and 2) a strain-gauge bridge placed at the base of the beam to measure the coupling torque. The physical parameters of the system are given in Table I. The strain signal is amplified by the dynamic strain amplifier (Kyowa DPM600) and filtered by a second-order Butterworth filter with cut-off frequency set to $300 \mathrm{~Hz}$. A National Instruments $6024 \mathrm{E}$ and a Measurement Computing CIO-DIO24 are used in a $\mathrm{PC}$ in conjunction with the Real Time Windows Target of MATLAB. The sampling time is set to $0.002 \mathrm{~s}$.

\section{SYSTEM MODEL}

Consider a flexible arm (see Fig. 2) composed of: a) a motor and a reduction gear of $1: n$ at the base with total inertia (rotor and hub) $J_{0}$, dynamic friction coefficient $\nu$ and Coulomb friction torque $\Gamma_{f}$; b) a flexible beam with uniform linear mass density $\rho$, uniform bending stiffness $E I$ and length $L$; and c) a payload of mass $M_{P}$ and rotational inertia $J_{P}$. Furthermore,

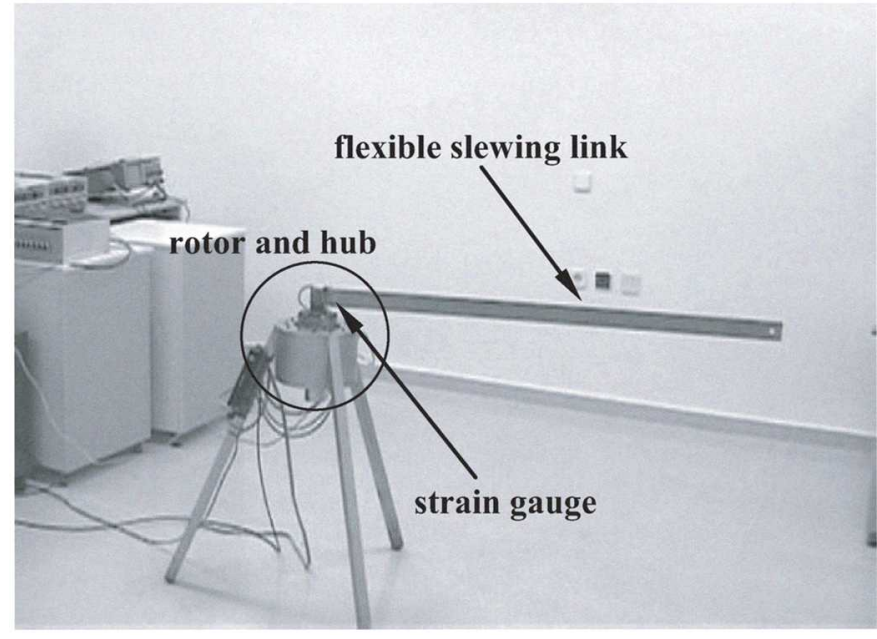

Fig. 1. Experimental platform.

\begin{tabular}{ccc}
\hline Stiffness $\left(\mathrm{Nm}^{2}\right)$ & $E I$ & 2.40 \\
\hline Width $(\mathrm{m})$ & $h$ & 0.05 \\
\hline Thickness $(\mathrm{m})$ & $b$ & 0.002 \\
\hline Length $(\mathrm{m})$ & $L$ & 1.26 \\
\hline Linear mass density $(\mathrm{kg} / \mathrm{m})$ & $\rho$ & 0.268 \\
\hline Mass of the beam $(\mathrm{kg})$ & $M_{b}$ & 0.338 \\
\hline Range of the payload mass $(\mathrm{kg})$ & $M_{P}$ & $0.0 \div 0.300$ \\
\hline Motor constant $(\mathrm{Nm} / \mathrm{V})$ & $K$ & 0.474 \\
\hline Reduction gear ratio & $n$ & 50 \\
\hline Inertia of the motor and hub $\left(\mathrm{kgm}^{2}\right)$ & $J_{0}$ & 0.79 \\
\hline
\end{tabular}

TABLE I

PARAMETERS OF THE FLEXIBLE ARM

the applied torque is $\Gamma_{m}, w(x, t)$ is the elastic deflection measured from the undeformed beam, $\theta_{m}$ is the joint angle and $\theta_{t}$ is the tip angle. In order to deduce the equations of the dynamic model, the pseudo-clamped configuration (see [23]) is utilized. Thus, the non-inertial frame $(x, y)$ rotates with the motor and the overall structure rotates in an inertial frame $(\mathrm{X}, \mathrm{Y})$.

The equation of the momentum balance at the output side

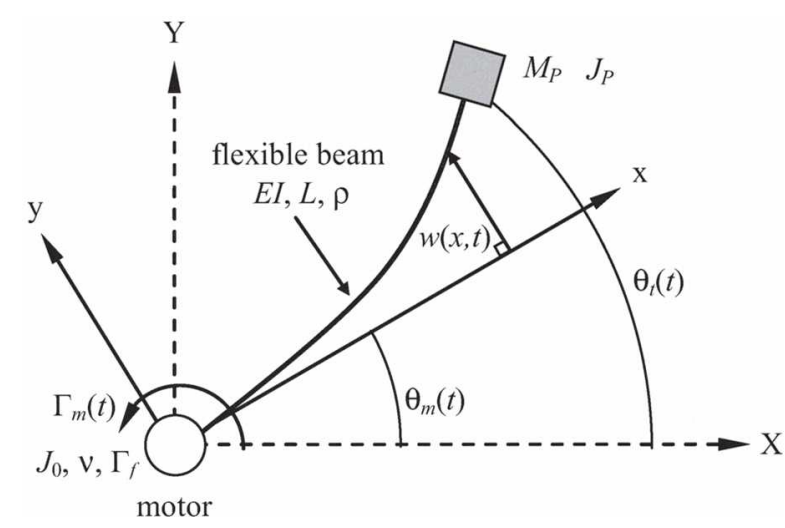

Fig. 2. Parametric representation of the flexible arm system 
of the gear is given by:

$$
\Gamma_{m}(t)=n K V(t)=J_{0} \ddot{\theta}_{m}(t)+\nu \dot{\theta}_{m}(t)+\Gamma_{\text {coup }}(t)+\Gamma_{f}(t),
$$

where $V$ is a voltage that controls the motor; $K$ is a constant that relates the motor torque $\left(\Gamma_{m}\right)$ and the control voltage $(V)$; and $\Gamma_{\text {coup }}$ is the coupling torque in the joint due to the link and the payload, and can be obtained using the formula:

$$
\Gamma_{\text {coup }}(t)=E I w^{\prime \prime}(0, t),
$$

where $w^{\prime \prime}(0, t)$ is proportional to the strain measured at the base of the link.

The dynamic behavior of the link inclusive of the payload can be modeled as an Euler-Bernoulli beam with the corresponding boundary conditions to account for the payload. Thus, a boundary value problem for the undamped free vibration can be written as:

$$
E I w^{\prime \prime \prime \prime}(x, t)+\rho\left(x \ddot{\theta}_{m}(t)+\ddot{w}(x, t)\right)=0,
$$

subject to the four boundary conditions:

$$
\begin{array}{r}
w(0, t)=0, w^{\prime}(0, t)=0, \\
E I w^{\prime \prime}(L, t)=J_{P}\left(\ddot{\theta}_{m}(t)+\ddot{w}^{\prime}(L, t)\right), \\
E I w^{\prime \prime \prime}(L, t)=M_{P}\left(L \ddot{\theta}_{m}(t)+\ddot{w}(L, t)\right) .
\end{array}
$$

Proceeding in the same way as described in [23], the characteristic equation is obtained. The infinite number of solutions of this equation provides the natural frequencies of the vibration modes which correspond with the poles of transfer function whose input is the joint angle $\theta_{m}$. To carry out a modal analysis, the input to the system has to be zero, which means $\ddot{\theta}_{m}(t)=0$. Later, the method of separation of variables $w(x, t)=\phi(x) q(t)$ (one dependable on the temporal coordinate $q(t)$ and one dependable on the spatial coordinate $\phi(x)$ ) is applied to the boundary value problem defined by eqs. (3) and (4). This leads to two differential equations; a time-dependent one given by:

$$
\ddot{q}(t)+\omega^{2} q(t)=0,
$$

and a spatially-dependent one given by:

$$
\phi^{\prime \prime \prime \prime}(x) \quad \beta^{4} \phi(x)=0,
$$

with $\beta^{4}=\rho \omega^{2} / E I$ and $\omega$ being the frequency. Finally, the form of the solution of the spatial-dependent part

$$
\begin{gathered}
\phi(x)=A \sin (\beta x)+B \cos (\beta x) \\
+C \sinh (\beta x)+D \cosh (\beta x),
\end{gathered}
$$

in which $A, B, C$ y $D$ are constants, is substituted into the boundary conditions (4) and the following equations of the system are obtained:

$$
\left[\begin{array}{cccc}
0 & 1 & 0 & 1 \\
1 & 0 & 1 & 0 \\
q_{31} & q_{32} & q_{33} & q_{34} \\
q_{41} & q_{42} & q_{43} & q_{44}
\end{array}\right]\left[\begin{array}{l}
A \\
B \\
C \\
D
\end{array}\right]=\left[\begin{array}{l}
0 \\
0 \\
0 \\
0
\end{array}\right]
$$

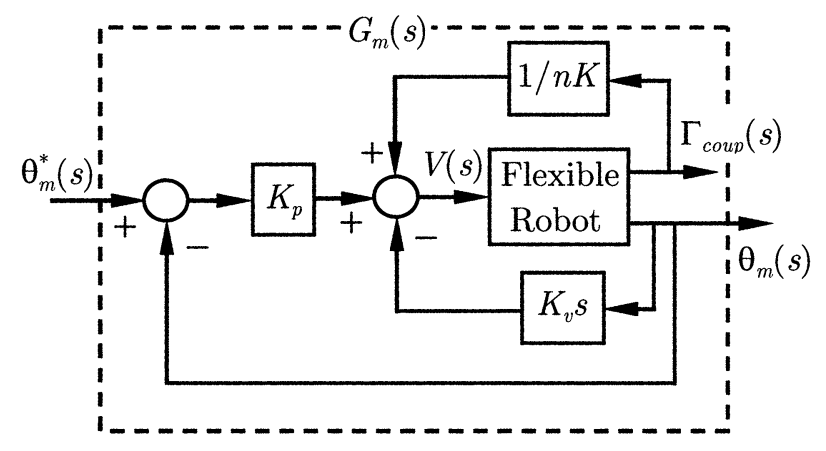

Fig. 3. Schematic of the inner control loop formed by a PD control of $\theta_{m}$ plus the decoupling term $1 / n K$

where $q_{31}=\left(\beta^{3} J_{P} / \rho\right) c_{L}+s_{L}, q_{32}=\left(\beta^{3} J_{P} / \rho\right) s_{L}+c_{L}$, $q_{33}=\left(\beta^{3} J_{P} / \rho\right) \operatorname{ch}_{L} \quad s h_{L}, q_{34}=\left(\beta^{3} J_{P} / \rho\right) s h_{L}+c h_{L}$, $q_{41}=\left(\beta M_{P} / \rho\right) s_{L} \quad c_{L}, q_{42}=\left(\beta M_{P} / \rho\right) c_{L}+s_{L}, q_{43}=$ $\left(\beta M_{P} / \rho\right) s h_{L}+c h_{L}$ and $q_{44}=\left(\beta M_{P} / \rho\right) c h_{L}+s h_{L}$, in which $c_{L}=\cos (\beta L), s_{L}=\sin (\beta L), c h_{L}=\cosh (\beta L)$ and $s h_{L}=\sinh (\beta L)$. To obtain a non-trivial solution of eq. (8), the determinant of the matrix of this equation has to be zero. This determinant is known as the characteristic equation and the infinite number of natural frequencies is obtained from its solutions.

\section{General CONTROL SCHEME}

The proposed control scheme comprises of two nested control loops. The first loop is a proportional derivative (PD) control of the joint angle $\theta_{m}$ plus a decoupling term to make the dynamics between $\theta_{m}$ and its reference $\theta_{m}^{*}$ independent of the link dynamics. The control voltage can be written as follows (see Fig. 3)

$$
\begin{aligned}
& V(t)=\Gamma_{\text {coup }}(t) / n K+K_{p}\left(\theta_{m}^{*}(t) \quad \theta_{m}(t)\right) \\
& K_{v} \dot{\theta}_{m}(t)
\end{aligned}
$$

where $\Gamma_{\text {coup }}(t) / n K$ (decoupling term) makes the design of the PD constants $\left(K_{p}, K_{v}\right)$ independent of the link dynamics. The substitution of (9) into (1) without considering $\Gamma_{f}$ yields

$$
\begin{aligned}
J_{0} \ddot{\theta}_{m}(t)+\left(n K K_{v}+\nu\right) \dot{\theta}_{m}(t) & +n K K_{p} \theta_{m}(t) \\
= & n K K_{p} \theta_{m}^{*}(t),
\end{aligned}
$$

which only depends on the motor dynamics. By tuning $K_{p}$ and $K_{v}$, we can design the dynamics of this closed loop system $G_{m}(s)$ (see Fig. 3). In addition, $G_{m}(s)$ is robust to Coulomb friction and to changes in the dynamic friction [24]. The system controlled by the first control loop is shown in Fig. 4.

The transfer functions $G_{1}(s)$ and $G_{2}(s)$ are lightly damped systems whose natural frequencies are the solutions of eq. (8). In addition, it was proved in [21] that $(1 / s) G_{2}(s)$ is strictly passive, i.e. its phase is between $90^{\circ}$ and $90^{\circ}$ (see Lemma 6.1 of [25]). Therefore, the phase of $\left(1 / s^{2}\right) G_{2}(s)$ is between $180^{\circ}$ and $0^{\circ}$ (like a collocated system) and can be modeled 


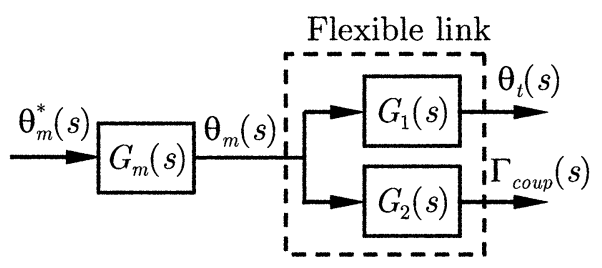

Fig. 4. System controlled only by the inner control loop

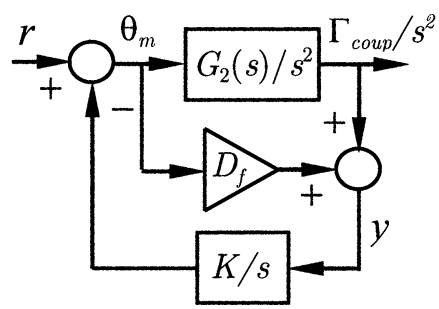

(a)

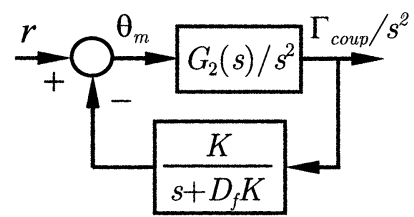

(b)

Fig. 5. (a) System $\left(1 / s^{2}\right) G_{2}(s)$ controlled by the IRC scheme proposed in [22]. (b) Equivalent representation of the IRC scheme where $D_{f}$ and $K$ are grouped into a lossy integrator.

as follows

$$
\frac{1}{s^{2}} G_{2}(s)=\sum_{i=1}^{N} \frac{\gamma_{i}}{s^{2}+2 \xi_{i} \omega_{i} s+\omega_{i}^{2}}+D,
$$

where $\omega_{i}$ is the natural frequency (solution of eq. (8)) and $\xi_{i}$ is the damping coefficient for the $i$ vibration mode, $\gamma_{i}>0 \forall i$, $N$ is the number of considered modes and $D$ is a feed-through term that models the effect of high-frequency modes on low frequency zeros due to the truncation [26]. Thus, the system $\left(1 / s^{2}\right) G_{2}(s)$ can be controlled by an IRC with the scheme illustrated in Fig. 5(a), [22]. An alternative representation of this control scheme is given in Fig. 5(b). The pole-zero map of the system is shown in Fig. 6(a). The feed-through term $\left(D_{f}<0\right)$, which is a negative real number, places a pair of low-frequency complex conjugate zeros between the origin and the first pair of complex conjugate poles of the system $\left(G_{2}\right)$ (see Fig. 6(b)). Thus, the system can be controlled with an integrator in positive feedback $(K<0)$, since the phase of $\left(K / s^{3}\right)\left(G_{2}(s)+D_{f}\right)$ lies between $90^{\circ}$ and $90^{\circ}$ (see Fig. $6(\mathrm{c}))$. In this work, the control scheme of Fig. 5(b) is chosen since it is more convenient for practical implementation (see reference [27]).

During practical implementation we must take into account that: a) the double integrator $\left(1 / s^{2}\right)$ is not possible in practice and b) the subsystem $G_{m}$ has a relative order of two and has the potential to deem the controlled system unstable. The

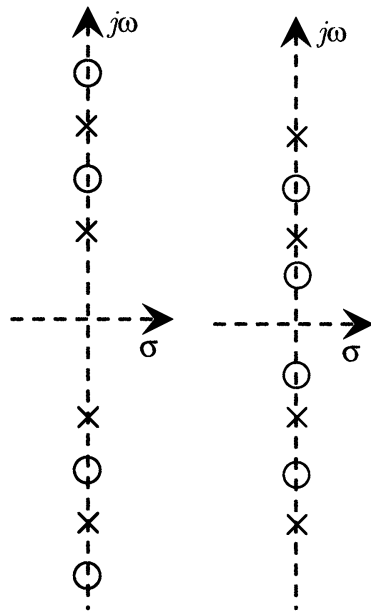

(a)

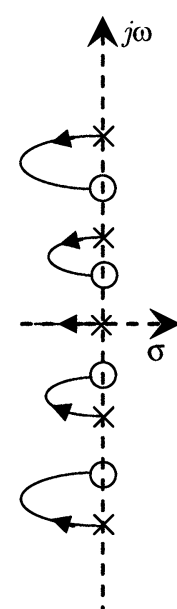

(c)
Fig. 6. (a) Pole-zero map of $\left(1 / s^{2}\right) G_{2}(s)$. (b) Pole-zero map of $\left(1 / s^{2}\right) G_{2}(s)+D_{f}$. (c) Root locus design of the parameter $\mathrm{K}$ for the IRC scheme of Fig. 5(a)

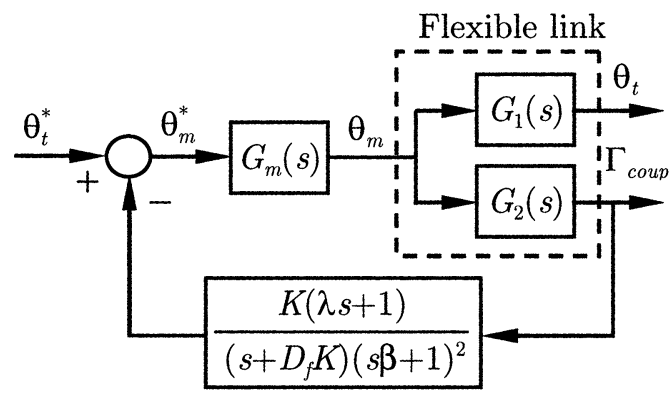

Fig. 7. Schematic of the IRC based inner-loop control strategy.

double integration is implemented by combining two lossy integrators with their combined transfer function given by $\left(1 /(s \beta+1)^{2}\right)$. The stability problem due to the relative order of $G_{m}$ can be solved by adding a zero to the outer controller. Thus, taking into account all the practical considerations, the equation of the outer controller is given by:

$$
C(s)=\frac{K(\lambda s+1)}{\left(s+D_{f} K\right)(\beta s+1)^{2}},
$$

where $\lambda$ places the zero that compensates the dynamics of $G_{m}$ and guarantees the stability of the overall system for high vibration modes (i.e., the phase of $G_{m}(s) G_{2}(s) C(s)$ is lies between $180^{\circ}$ and $0^{\circ}$ ). One of the advantages of this design is that the magnitude of $G_{m}(s) G_{2}(s) C(s)$ decreases with increasing frequency. Hence, it is robust to antialiasing filters (like the Butterworth filter of the strain dynamic amplifier). The next section presents the experimental results obtained on the single-link flexible manipulator.

\section{EXPERIMENTAL RESULTS}

The tuning of the parameters of the PD controller $\left(K_{p}\right.$, $K_{v}$ ) is carried out to achieve a critically damped second-order system. Thus, we have the following transfer function between 


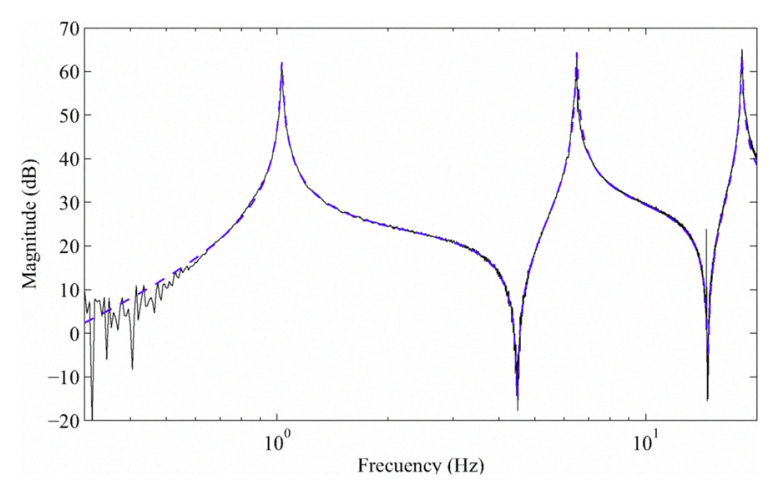

Fig. 8. Magnitude response of the measured ( - ) and modeled system (--) of $G_{2}(s)$

the motor angle and its reference

$$
\theta_{m}(s) / \theta_{m}^{*}(s)=1 /(1+\alpha s)^{2},
$$

where $\alpha$ is the constant time of $G_{m}(s)$. From eqs. (10) and (13) the values of $K_{p}$ and $K_{v}$ are obtained.

$$
K_{p}=J_{0} / n K \alpha^{2} ; K_{v}=\left(\begin{array}{ll}
2 J_{0} & v \alpha
\end{array}\right) / n K \alpha .
$$

We set $\alpha=0.01$, then from Table I and eq. (14), the values of $K_{p}$ and $K_{v}$ are 350.9 and 6.9. This value of $\alpha$ makes the transfer function $G_{m}(s)$ robust to Coulomb friction and does not saturate the DC motor. Identification for $G_{2}(s)$ with $M_{P}=0$ $\mathrm{kg}$ and $J_{P}=0 \mathrm{kgm}^{2}$ is carried out. The frequency response of $G_{2}(s)$ is obtained by applying a chirp signal to the joint angle reference of amplitude equal to $0.05 \mathrm{rad}$. Using a subspacebased system identification technique, an accurate model of the experimental system was obtained [28]. This transfer function captures the system dynamics within the bandwidth of interest, with sufficient accuracy and can be written as:

$$
\begin{array}{r}
\frac{1}{s^{2}} G_{2}(s)=\frac{13.74}{s^{2}+0.07 s+41.9}+\frac{14.33}{s^{2}+0.36 s+1668} \\
\frac{12.12}{s^{2}+0.94 s+1.3 \cdot 10^{4}}+0.001 .
\end{array}
$$

The frequency response functions (FRF) of the measured and modeled system of $G_{2}(\mathrm{~s})$ are plotted in Fig 8.

For the unloaded experimental platform, $M_{P}=0 \mathrm{~kg}$ and $J_{P}=0 \mathrm{kgm}^{2}$. The dynamics of $G_{2}$ and $G_{m}$ correspond with the eq. (15) and $1 /(0.01 s+1)^{2}$ respectively. The controller parameters are tuned in a stepwise fashion described as follows.

- Place the poles of the double integrator and choose the bandwidth for the IRC. There is a tradeoff between the bandwidths of $1 /(s / \beta+1)^{2}$ and $\left(K /\left(s+D_{f} K\right)\right)$, since a high bandwidth of $1 /(s / \beta+1)^{2}$ allows us to increase the rise time in $\theta_{m}$ but limits the imparted damping by the IRC $\left(K /\left(s+D_{f} K\right)\right)$. The practical rule to guarantee the stability of the closed loop system is that the total phase of $1 /(s / \beta+1)^{2}$ and $\left(K /\left(s+D_{f} K\right)\right)$ must be $180^{\circ}$ in $\omega_{1} / 2$, where $\omega_{1}$ is the natural frequency of the first vibration mode.

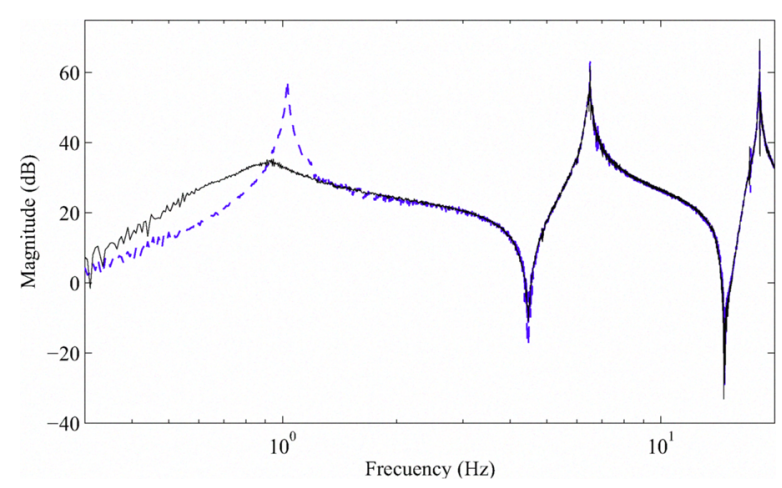

Fig. 9. Magnitude response of $G_{m}(s) G_{2}(s)$ (-) and the closed loop system between coupling torque and tip angle reference (see Fig. 7) (--)

- Tune the value of $\lambda$ to guarantee that the phase of $(\lambda s+$ 1) $G_{m}(s)$ lies between $90^{\circ}$ and $0^{\circ}$.

- Tune the gain of $C(s)\left(1 / D_{f}\right)$ by using the root locus technique.

The proposed outer controller after following the stepwise fashion is $\beta=0.5, D_{f} K=2, \lambda=1 / 50$ and $1 / D_{f}=0.9$.

Fig. 9 shows the magnitude response of the open loop $\left(G_{m}(s) G_{2}(s)\right)$ and the closed loop. The first three modes are attenuated by $23 \mathrm{~dB}, 2 \mathrm{~dB}$ and $0 \mathrm{~dB}$ respectively. The low performance of the outer controller in the second and third vibration mode is due to the small bandwidth of IRC (i.e., small value of $D_{f} K$ ). A better damping can be obtained if we increase the values of $\beta$ and $D_{f} K$. However, the time to reach the final position is also increased. The reference of the time response is a Bezier polinomial of fourth order with final value $0.5 \mathrm{rad}$ and the trajectory time of $1 \mathrm{~s}$. The two represented variables are the estimated tip angle and the coupling torque measured at the base of the arm. The estimation of the tip angle is made with a full-order observer whose inputs are the motor angle and the coupling torque. Such a full-order observer is only used to estimate the system output for the comparison, and is not used for control purposes. Fig. 10 shows the reference and the estimated tip angle without and with control. In this figure, it can be observed that the system reaches the final position without vibration. Fig. 11 shows the coupling torque without and with control. In this figure, it can observed that there is more than one vibration mode in the time response and the damping that is imparted by the controller.

\section{CONCLUSION}

A new approach for the control of flexible manipulators based on the IRC scheme has been proposed in this work. The control scheme consists of two control loops. The first loop controls the joint position independent of the link dynamics. Thus, high control gains or integral actions can be implemented without affecting the stability of the outer loop. The outer loop imparts damping to the overall system and guarantees stability.

This work presents the following characteristics (i) the design of the two control loop is independent of the relationship between link and joint parameters, which allows us to use high 


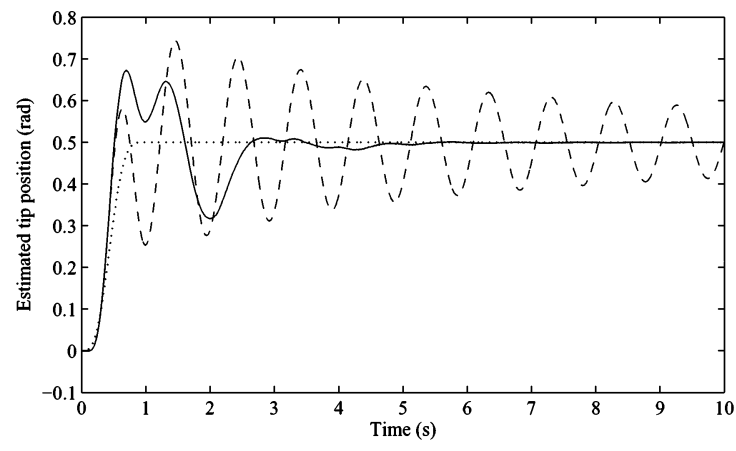

Fig. 10. Time response of the estimated tip angle obtained from experimenta data. Reference (...) and estimated tip position in open loop (- -) and closed loop (-)

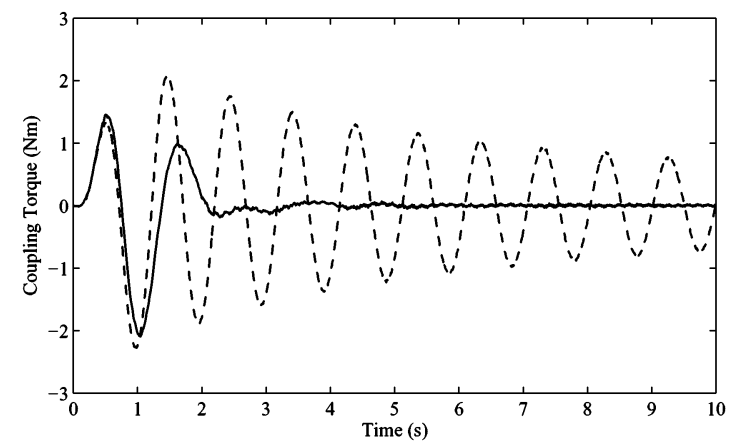

Fig. 11. Time response of the coupling torque in open loop (- -) and closed loop (-)

gains or integral actions that make the system robust to joint frictions, (ii) both the designs of the control loops and the stability analysis are simple and effective, (iii) reducing gears can be used and (iv) it is not sensitive to low frequencies and is more robust to unmodeled dynamics like antialising filters. Thus, this approach is more suitable for real implementation than other control methodologies based on minimum phase systems mentioned in the Introduction. The next step is to extend this work to multi-link flexible manipulators.

\section{ACKNOWLEDGMENT}

The authors would like to thank Dr I. M. Díaz for his help. This work has been supported by the Spanish Government Research Program with the projects DPI2006-13593 and DPI2003-03326 (MCyT).

\section{REFERENCES}

[1] F. Wang and Y. Gao, Advanced studies of flexible robotic manipulators, modeling, design, control and applicattions. World Scientific, New Jersey (EUA), 2003.

[2] M. Benosman and G. Vey, "Control of flexible manipulators: A survey," Robotica, vol. 22, pp. 533-545, 2004

[3] S. K. Dwivedy and P. Eberhard, "Dynamic analysis of flexible manipulators, a literature review," Mechanism and Machine Theory, vol. 41, no. 7, pp. 749-777, 2006.

[4] A. R. Fraser and R. W. Daniel, Perturbation techniques for flexible manipulators. Kluwer Academic Publisher, 1991.

[5] R. H. Canon and E. Schmitz, "Initial experiments on the end-point control of a flexible robot," International Journal on Robotics Research vol. 3, no. 3, pp. 62-75, 1984.
[6] V. Etxebarria, A. Sanz, and I. Lizarraga, "Real-time experimental contro of a flexible robotic manipulator using a composite approach," in Proceedings of the IEEE International Conference on Control Applications, September 2004, pp. 955-960.

[7] T. Kotnick, S. Yurkovich, and U. Ozguner, "Acceleration feedback control for a flexible manipulator arm," Journal of Robotic System, vol. 5, no. 3, pp. 181-196, 1998.

[8] A. De Luca, P. Lucibello, and G. Ulivi, "Inversion techniques of trajectory control of flexible robot arm," Journal of Robotic Systems, vol. 6 , no. 4 , pp. $325-344,1989$

[9] S. O. R. Moheimani and R. L. Clark, "Minimizing the truncation error in assumed modes models of structures," Transactions of the ASME, Journal of Vibration \& Acoustics, vol. 122, no. 3, pp. 332-335, July 2000.

[10] T. C. Yang, J. C. S. Yang, and P. Kudva, "Load adaptive control of a single-link flexible manipulator," IEEE Transactions on Systems Management Cybernet, vol. 22, no. 1, pp. 85-91, 1992.

[11] D. Farruggio and L. Menini, "Two degrees of freedom h control of a flexible link," in Proceedings of the American Control Conference, June 2000, pp. 2280-2284.

[12] M. Karkoub and K. Tamma, "Modelling and mu-synthesis control of flexible manipulators," Computers and Structures, vol. 79, pp. 543-551, 2001.

[13] Y. P. Chen and H. T. Hsu, "Regulation and vibration control of an fembased single-link flexible-arm using sliding-mode theory," Journal of Vibration and Control, vol. 7, no. 5, pp. 741-752, 2001.

[14] Z. Su and K. A. Khorasani, "Neural-network-based controller for a single-link flexible manipulator using the inverse dynamics approach," IEEE Transactions on Industrial Electronics, vol. 48, no. 6, pp. 1074 1086, 2001.

[15] V. G. Moudgal, W. A. Kwong, K. M. Passino, and S. Yurkovich, "Fuzzy learning control for a flexible-link robot," IEEE Transactions on Fuzzy System, vol. 3, no. 2, pp. 467-488, 2002.

[16] D. Wang and M. Vidyasagar, "Passive control of a stiff flexible link," The International Journal of Robotics Research, vol. 11, no. 6, pp. 572-578, 1992.

[17] L. Liu and K. Yuan, "Noncollocated passivity-based pd control of a single-link flexible manipulator," Robotica, vol. 21, no. 2, pp. 117-135, 2003.

[18] Z. H. Luo, "Direct strain feedback control of flexible robot arms: new theoretical and experimental results," IEEE Transactions on Automatic Control, vol. 38, no. 11, pp. 1610-1622, 1993.

[19] Z. H. Luo and D. X. Feng, "Nonlinear torque control of a single-link flexible robot." Journal of Robotic System, vol. 16, no. 1, pp. 25-35, 1999.

[20] I. A. Mahamood, S. O. R. Moheimani, and B. Bhikkaji, "Precise tip positioning of a flexible manipulator using resonant control," in IEEE/ASME International Conference on Advanced Intelligent Mechatronics, 2007.

[21] E. Pereira, I. M. Díaz, J. J. Cela, and V. Feliu, "A new methodology for passivity based control of single-link flexible manipulator," in IEEE/ASME International Conference on Advanced Intelligent Mechatronics, 2007.

[22] S. S. Aphale, A. J. Fleming, and S. O. R. Moheimani, "Integral resonant control of collocated smart structures," Smart Mater. Struct., vol. 16, pp. 439-446, 2007.

[23] F. Bellezza, L. L. L, and G. Ulivi, "Exact modelling of the flexible slewing link," Proceedings of the IEEE International Conference on Robotics and Automation, vol. 1, pp. 734-804, May 1990.

[24] V. Feliu, D. S. Rattan, and H. B. Brown, "Control of flexible arms with friction in the joints," IEEE Transactions on Robotics and Automation, vol. 9, no. 4, pp. 467-475, 1993.

[25] H. K. Khalil, Nonlinear Systems. New York: Macmillan, 1992.

[26] R. L. Clark, "Accounting for out-of-bandwidth modes in the assumed modes approach: implications on collocated output feedback control,' Transactions of the ASME, Journal of Dynamic Systems, Measurement, and Control, vol. 119, pp. 390-395, 1997.

[27] E. Pereira, S. S. Aphale, and S. O. R. Moheimani, “Op-amp based analog implementation of the integral resonant control scheme," in International Conference on Adaptive Structures and Technologies (Accepted), 2008.

[28] T. McKelvey and L. Akay, H.and Ljung, "Subspace based multivariable system identification from frequency response data," IEEE Transactions on Automatic Control, vol. 41, pp. 960-978, 1996. 